\title{
MOND's acceleration scale as a fundamental quantity
}

\author{
Tula Bernal ${ }^{1}$, Salvatore Capozziello ${ }^{2,3}$, Gerardo Cristofano ${ }^{2,3}$, Mariafelicia De Laurentis ${ }^{2,3}$ \\ ${ }^{1}$ Instituto de Astronomía, Universidad Nacional Autónoma de México, \\ AP 70-264, Distrito Federal 04510, México, ${ }^{2}$ Dipartimento di Scienze Fisiche, \\ Università di Napoli "Federico II", Napoli, Italy, ${ }^{3}$ INFN Sez. di Napoli, \\ Compl. Univ. di Monte S. Angelo, Edificio G, Via Cinthia, I-80126, Napoli, Italy
}

(Dated: March 20, 2018)

\begin{abstract}
Some quantum-cosmic scaling relations indicate that the MOND acceleration parameter $a_{0}$ could be a fundamental quantity ruling the self-gravitating structures, ranging from stars and globular clusters up to superclusters of galaxies and the whole observed universe. We discuss such coincidence relations starting from the Dirac quantization condition ruling the masses of primordial black holes.
\end{abstract}

PACS numbers: 04.50.+h, 95.36.+x, 98.80.-k

Keywords: Alternative theories of gravity; cosmology; large scale structure; quantization conditions.

\section{INTRODUCTION}

The observed scales of astrophysical self-gravitating systems, like galaxies, galaxy clusters and the universe itself, constitute a puzzle of modern physics. From a fundamental point of view, it is expected that all bounded self-gravitating structures have a common origin related to the cosmic microwave background radiation. Such structures should bring the signature of primordial quantum fluctuations which should have determined the scales of systems that we observe today.

The main goal of studies dealing with this issue is to frame the large scale structure into some unifying theory in which all the today observed structures can be treated under the same fundamental standard. In this sense, the remnants of the primordial epochs, probed by the cosmological observations, are the ideal test to constrain such theories.

Looking for a fundamental theory connecting the microscopic to the macroscopic scales, many "coincidences" have been identified among cosmic and fundamental parameters, like the cosmological constant, the gravitational constant, the speed of light and the Planck constant. The so-called Large Numbers Coincidence (LNC) [1], for example, refers to some fundamental relations to pure numbers of order $10^{40}$. Eddington and Dirac hypothesized that such coincidences could be dynamically generated by the cosmological parameters related to quantum ones. Furthermore, this coincidence seems to to occur at the same epoch in which other coincidences related to cosmic parameters occur. This fact suggests some underlying physical law that should be fully understood [2].

In addition, there is the issue involving the so-called "dark" components, i.e. dark matter and dark energy, being approximately the $95 \%$ of the total mass-energy content of the universe. In principle, the dark matter was postulated to explain the observed rotation curves of spiral galaxies [3]; then it was necessary to explain the mass to light ratios of galaxies and galaxy clusters, the gravitational lensing, the structure formation and most of astrophysical phenomena and structures that escape the standard description by Einstein (Newton) gravity and luminous matter [4], On the other hand, the dark energy has been postulated in order to explain the today observed accelerated expansion rate of the Hubble cosmic fluid, firstly deduced by the supernovae Ia observations [5, 6].

As alternatives to the dark matter and energy problems, several theories modifying the gravity, instead of the inclusion of unknown components, have been proposed, in both non-relativistic and relativistic regimes, indicating a failure in the Newtonian and general relativistic theories of gravity at large scales (see e.g. 7, 8] ).

In the non-relativistic limit, Milgrom introduced the MOdified Newtonian Dynamics (MOND) [9 11] to explain the observed rotation curves of spiral galaxies, through the inclusion of a phenomenological acceleration scale $a_{0}$ such that, for accelerations much greater than $a_{0}$, the dynamics is Newtonian and for accelerations much less than $a_{0}$, the dynamics is modified and reduces to constant circular velocities at large radii.

The MOND frame has been proved to be successful on many astrophysical situations, but difficult on others (see e.g. [8] and references therein). Despite these facts, the key feature of this modification, is the introduction of an acceleration scale $a_{0}$ into the gravitational interaction [4]. Concerning $a_{0}$, some of the mentioned coincidences can be considered involving the Hubble constant, the cosmological constants, and the the nucleon size [2]. Quantum mechanics and quantum field theory play a main role in understanding such coincidences showing that $a_{0}$ could be actually considered among the number of fundamental parameters of physics.

In this paper, we will face this problem. Starting from a quantum field theory approach, a quantum relation for primordial black holes has been proposed whose validity extends from the Planck scales to the today observed astrophysical structures [12]. It can be proven that such a quantum relation is a scaling one containing, in principle, the signature of astrophysical structures starting from their basic constituents, the nucleons [13]. Specifically, it reproduces the so-called Eddington-Weinberg relation [14], as well as the phenomenological statistical hy- 
pothesis for self-gravitating systems [15, 16], where the characteristic sizes of astrophysical structures can be recovered assuming that gravity is the overall interaction assembling systems from fundamental microscopic constituents.

In this paper, we show how, starting from fundamental scaling relations involving cosmological coincidences, MOND's acceleration emerges as a characteristic scale for self-gravitating astrophysical systems. We take into account only characteristic masses, radii and the baryonic components. It is worth noticing that the acceleration scale is addressed without assuming any dark component.

The paper is organized as follows. In Sec. III we recall the scaling hypothesis working, in principle, for all selfgravitating structures, from Planck's scales to the universe itself. From the scaling relations, a characteristic acceleration is deduced and identified as $a_{0}$ in terms of fundamental quantities.

In Sec. III, the signature of scaling relations for several self-gravitating systems is discussed as a consistency check to obtain the characteristic sizes of the structures from their characteristic baryonic masses.

Sec. IV is devoted to a brief summary on how the acceleration scale $a_{0}$ modifies the Newtonian dynamics. In Sec. V we discuss the coincidence relations showing that $a_{0}$ can be considered among the fundamental parameters of scaling relations.

Discussion and conclusions are reported in Sec. VI

\section{SCALING HYPOTHESIS FOR ASTROPHYSICAL SYSTEMS}

Assuming the Dirac quantization condition [17] and a quantum field theory result relating the electric and magnetic charges of primordial black holes to their masses $M$, the following quantization relation can be obtained [12]:

$$
G M^{2}=n \hbar c,
$$

where $G$ is the gravitational constant, $n$ is the quantization number and $\hbar$ is the reduced Planck constant. For $n=1$, the lowest mass allowed for a quantum black hole is the Planck mass.

Moreover, it can be shown that the above relation is valid for self-gravitating astrophysical structure, from globular clusters to the observed universe itself [12], and numerical agreement has been found with the statistical hypothesis (based on phenomenological considerations) applied to self-gravitating systems, defined as bound states with a very large number $N_{\text {as }}$ of constituents [15, 16]. This connection can be equivalently obtained either by considering the protons as the elementary constituents or, as usual in astrophysics, by considering stars as the granular components of galaxies, and the galaxies the granular components of galaxy clusters and superclusters ("granular approximation").
In this context, the working hypothesis is that the total action of the astrophysical self-gravitating system, $A_{\text {as }}$, can be achieved from the Planck constant as

$$
A_{\mathrm{as}} \approx \hbar N_{\mathrm{as}}^{3 / 2} .
$$

This means that the total action is given by $A_{\text {as }}=n_{\text {as }} \hbar$, where $n_{\text {as }}$ is the value of the quantum level number appearing in Eq. (11). By comparing this number with the corresponding value $N_{\mathrm{as}}^{3 / 2}$ from Eq. (2), a numerical scaling with characteristic sizes of globular clusters, galaxies, galaxy clusters and the observed universe can be achieved [18.

However, it is interesting to note that the quantization relation (11) scales "analytically" for any astrophysical system down to the Planck mass. In fact, the quantum relation can be rewritten in a more suitable form as 13.

$$
G M_{\mathrm{as}}^{2}=\left(\frac{N_{\mathrm{as}}}{N_{\mathrm{BH}}}\right)^{2} \hbar c,
$$

where $N_{\mathrm{BH}}$ is the number of protons in primordial black holes.

Writing the mass of the self-gravitating system considering its number of protons as

$$
M_{\mathrm{as}} \approx N_{\mathrm{as}} m_{p},
$$

one gets simply back $G M_{\mathrm{Pl}}^{2}=\hbar c$, and the scaling hypothesis (2) is immediately verified [12].

Another interesting outcome obtained by quantization relation (11), can be achieved by using the relation

$$
\frac{G M_{\mathrm{as}}}{R_{\mathrm{as}}^{2}} \equiv 2 \pi a_{*},
$$

where $R_{\text {as }}$ is the characteristic radius of the astrophysical structure and the acceleration $a_{*}$ can be shown to be a universal constant.

In fact, it is possible to derive the size of the astrophysical structure in terms of the number of its fundamental constituents (protons), $N_{\text {as }}$, and the fundamental scale of the proton, the Compton wavelength $\lambda_{p}$. It is

$$
R_{\mathrm{as}} \approx 10 \sqrt{N_{\mathrm{as}}} \lambda_{p} .
$$

Such a result reproduces the statistical hypothesis relation proposed in Refs. [15, 16] with a correcting factor of order 10 coming from statistical uncertainties [13].

By using the relation (4) for the mass $M_{\text {as }}$ and the size $R_{\text {as }}$ from Eq. (6), we can rewrite the scaling relation (5) as

$$
\frac{G M_{\mathrm{as}}}{R_{\mathrm{as}}^{2}}=\frac{G m_{p}}{\left(10 \lambda_{p}\right)^{2}} \equiv 2 \pi a_{*} .
$$


Also, by considering the case of the observed universe and using the Raychaudhuri equation [19], the last relation can be written as

$$
\frac{G M_{\mathrm{u}}}{R_{\mathrm{u}}^{2}}=\frac{c^{2}}{R_{\mathrm{u}}}=2 \pi a_{*} .
$$

It is interesting to notice that specializing Eq. (6) to the radius of the universe and using Eqs. (4) and (8), the Eddington-Weinberg relation for the radius of the universe is easily obtained with the correcting factor 10 :

$$
h=\frac{1}{10} \sqrt{G R_{\mathrm{u}} m_{p}^{3}} .
$$

In addition, from Eq. (7), the value of the acceleration $2 \pi a_{*}$ is found to be

$$
2 \pi a_{*}=\frac{G m_{p}^{3} c^{2}}{(10 h)^{2}}
$$

which is a constant. Evaluating $a_{*}$ in the last equation, we find the value $a_{*} \approx 1.01 \times 10^{-10} \mathrm{~m} / \mathrm{s}^{2}$.

It is important to stress that the equation for the constant acceleration is valid for any astrophysical system where Eq.(6) gives its characteristic radius and Eq.(4) gives its mean mass.

As we will see, the derived value for $a_{*}$ is very close to the phenomenological value obtained for the constant acceleration scale introduced in the MOND theory, that is $a_{*} \approx a_{0}$. However the value of $a_{*}$ has been obtained here combining the fundamental quantities $G, h, c$ and $m_{p}$. The meaning of this result is that the acceleration $a_{0}$ could be a fundamental quantity as we want to show in the discussion below.

\section{ASTROPHYSICAL SYSTEMS FROM SCALING RELATIONS}

A self-gravitating astrophysical system is a gravitationally bounded assembly of stars and gas, and other point masses that can be neglected with respect to the main stellar and gas content. These systems vary over more than fourteen orders of magnitude in size and mass, from individual stars to star clusters containing $10^{2}$ to $10^{6}$ stars, through galaxies containing $10^{5}$ to $10^{12}$ stars, to vast clusters containing thousands of galaxies [4].

The properties of these self-gravitating structures can be deduced by assuming them to be relaxed and virialized systems where gravity is the only overall interaction [4]. First, we define globular clusters, galaxies and clusters of galaxies, considering their typical lengths and masses. For the characteristic mass we will only take the baryonic one and, as the boundary ("size") of these systems is not univocally defined, we will suppose a characteristic length for the baryonic mass gravitationally bounded to the system. It is important to remark that we are not assuming a dark matter component for the systems. For example, for a spiral galaxy, we take a typical radius of $\sim 10 \mathrm{kpc}$, which is the typical radius for the visible disk, not $\sim 100 \mathrm{kpc}$ for the hypothetical dark matter halo.

Now, a star is not a purely self-gravitating system since, inside it, gravity is balanced by the pressure due to electromagnetic and nuclear interactions. However, we can find a characteristic gravitational length for the gravitating objects around a star (as the size of a "planetary system" around it). Of course, this depends on the environment in which the star is embedded, but we can assume a typical one, as the Solar neighborhood [4].

To obtain the characteristic interaction lengths for the systems, we are using the granular approximation, considering the stars as the granular constituents of the astrophysical structures, whose main constituents are the protons.

The globular clusters are very massive stellar systems, containing up to $10^{6}$ stars $\left(M_{\mathrm{gc}} \sim 10^{6} \mathrm{M}_{\odot}\right)$. The typical radii are of the order $\sim 10 \mathrm{pc}$. These systems are assumed completely virialized due to collisional interactions between stars [4].

A galaxy is a collisionless gravitating system, with masses ranging from $M_{\text {gal }} \sim 10^{8} \div 10^{9} \mathrm{M}_{\odot}$, for dwarf galaxies, and $M_{\text {gal }} \sim 10^{10} \div 10^{12} \mathrm{M}_{\odot}$, for giant galaxies. The sizes are not well defined since there is no effective boundary for the systems. Astronomers define operative characteristic sizes as the effective radius, $R_{e}$, containing half of the total luminosity or the tidal radius, $R_{t}$, defined as the distance from the center where the density drops to zero [4]. Assuming as a typical interaction length $R_{\text {gal }} \sim 1 \div 10 \mathrm{kpc}$ is quite reasonable for systems ranging from dwarf to giant galaxies.

Groups of galaxies are systems containing $10 \div 20$ galaxies, but they are not considered self-gravitating systems because they are always part of more extended selfgravitating structures (clusters of galaxies). For example, our Local Group is a part of the Virgo Cluster.

The clusters of galaxies are the largest self-gravitating structures in the universe. The biggest clusters have masses $\sim 10^{15} \mathrm{M}_{\odot}$ within $\sim 1 \mathrm{Mpc}$ from their centers.

The superclusters of galaxies are not considered effective self-gravitating systems because of their large sizes. For this reason, they are considered as expanding with the expansion rate of the universe. It is supposed that all the clusters of galaxies are part of a larger supercluster.

Finally, the mean number of atoms (baryons) in the observable universe is supposed to be $\sim 10^{80}$, corresponding to a mass of the order $M_{\mathrm{u}} \sim 10^{23} \mathrm{M}_{\odot}$. Furthermore, the estimated size for the observed universe vary between $R_{\mathrm{u}} \sim 10^{25} \div 10^{27} \mathrm{~m}$.

Let us now show how the scaling relation (6) holds for astrophysical structures. To this purpose, we write the mean radius $R_{\mathrm{as}}$, coming from Eq. (6), in terms of the mean baryonic mass $M_{\text {as }}$, given by Eq. (4) for the given astrophysical structure, as 


$$
R_{\mathrm{as}}=10\left(\frac{M_{\mathrm{as}}}{m_{p}^{3}}\right)^{1 / 2} \frac{h}{c} .
$$

From this relation, we obtain, for a globular cluster with mean mass $M_{\mathrm{gc}} \sim 10^{6} \mathrm{M}_{\odot}$, the characteristic radius $R_{\mathrm{gc}} \sim 15 \mathrm{pc}$.

For a typical giant galaxy with mean mass $M_{\text {gal }} \sim$ $10^{11} \mathrm{M}_{\odot}$, the corresponding characteristic radius is $R_{\text {gal }} \sim 5 \mathrm{kpc}$.

For a cluster of galaxies with mean mass $M_{\mathrm{cg}} \sim 5 \times$ $10^{14} \mathrm{M}_{\odot}$, we have a characteristic radius of $R_{\mathrm{cg}} \sim 0.5$ Mpc.

For the universe, assuming the mean mass $M_{\mathrm{u}} \sim$ $10^{23} \mathrm{M}_{\odot}$, we obtain the mean radius $R_{\mathrm{u}} \sim 10^{26} \mathrm{~m}$.

In this sense, we can calculate the corresponding characteristic interaction radius of a star with mean mass $M_{\text {star }} \sim 1 \mathrm{M}_{\odot}$, as $R_{\text {star }} \sim 3 \times 10^{3} \mathrm{au}$, where "au" is the astronomic unit.. This radius corresponds to the radius of the Oort cloud, which defines the typical outest gravitational boundary of our Solar System.

For the proton, we have that its characteristic interaction radius, from the scaling relation (6), is given by $R_{\mathrm{p}} \approx 10 \lambda_{p}$, and its characteristic acceleration scales as Eq. (7).

\section{THE MOND ACCELERATION PARAMETER}

The acceleration constant $a_{0}$ has been phenomenologically introduced by Milgrom [9] as a cut-off parameter in the MOND theory to discriminate between Newtonian gravity and modified dynamics. MOND is constructed to obviate the need of dark matter when applied to galactic systems, for which the standard Newtonian dynamics is a good approximation only for accelerations much larger than $a_{0}$, and the so-called deep MOND regime is valid for accelerations much less than $a_{0}$.

The best value for $a_{0}$ is obtained from the fit to the rotation curves of spiral galaxies in the deep MOND regime, in the vicinity of our Galaxy and for a value for the Hubble constant $H_{0}=75 \mathrm{~km} \mathrm{~s}^{-1} \mathrm{Mpc}^{-1}$, as $a_{0} \approx 1.2 \times 10^{-10} \mathrm{~m} / \mathrm{s}^{2}[20]$. This value is taken as a constant of the theory for all the applications to the different systems in the universe.

At this stage, MOND is purely phenomenological, and people has done many efforts to construct a fundamental theory from which MOND would be the correct limit for the accelerations $a \ll a_{0}$ (see e.g. [8] and references therein).

In the search of such fundamental theory, Milgrom first noticed the following coincidence between the value of the acceleration scale $a_{0}$, the Hubble constant at the present epoch and the speed of light [9, 10]:

$$
2 \pi a_{0} \approx c H_{0} .
$$

From the Friedmann - Lemaître - Robertson - Walker cosmological equations, the last relation can be written in terms of the cosmological constant $\Lambda$ as (see e.g. [10])

$$
2 \pi a_{0} \approx c\left(\frac{\Lambda}{3}\right)^{1 / 2}
$$

since $H_{0} \approx(\Lambda / 3)^{1 / 2}$ at the present time.

This fact can be interpreted, from one side, as the coincidence connecting MOND to cosmology and dark energy, and, on the other side, as the influence of the cosmic large scales to the local dynamics 10,11 .

An additional comment to this coincidence is the fact that, as the Hubble parameter $H_{0}$ varies with cosmic time, $a_{0}$ varies too. This is not necessarily the case since $a_{0}$ could be related to $\Lambda$ with the latter being constant. In this case, however, the problem of the tiny value of $\Lambda$ with respect to the large value of gravitational vacuum state still remains (the so-called Cosmological Constant Problem). Interestingly, variations of $a_{0}$ could induce secular evolution in galaxies and other galactic systems [10]. Although the value obtained from the rotation curve of a spiral galaxy, at the redshift $z=2.38$, is consistent with the local measured value from the best studied rotation curves of spiral galaxies in vicinity of the Milky Way, this possibility is not completely discarded because of the high uncertainties in the observations [10]. If $a_{0}$ does not vary with the cosmic time, then this coincidence just occurs at the present epoch.

\section{V. "COINCIDENCES" FOR THE VALUE OF $a_{0}$}

Assuming $a_{*}=a_{0}$, the scaling relation (5) holds for two very different systems: the observed universe and the nucleon.

In the case of the universe, it is possible to construct a length scale $l_{0}$ from the constants $G, c$ and $a_{0}$, such that $l_{0} \equiv c^{2} / a_{0} \approx 10^{27} \mathrm{~m}$, and a mass scale $M_{0} \equiv \mu_{0} c^{4} \approx 6 \times 10^{23} \mathrm{M}_{\odot}$ (see e.g. [10]). Looking for a connection with quantum theory, these scales can be seen as the Planck length and mass constructed from the fundamental constants $\hbar, G$ and $c$. In this sense, $l_{0}$ and $M_{0}$ can give the scales where MOND effects, combined with gravity, are expected. In fact, the Hubble radius and the mass of the universe can be written as

$$
\begin{gathered}
R_{H} \equiv \frac{c}{H_{0}} \approx \frac{l_{0}}{2 \pi} \sim 10^{26} \mathrm{~m}, \\
M_{\mathrm{u}} \approx \frac{c^{3}}{G H_{0}} \approx \frac{M_{0}}{2 \pi} \sim 10^{23} M_{\odot} .
\end{gathered}
$$

From these two relations, the coincidence (12) can be written as [10] 


$$
2 \pi a_{0} \approx \frac{G M_{u}}{R_{H}^{2}} .
$$

We can see that this result is exactly reproduced by Eq. (8) because, despite of the different definitions for the radius of the universe, the corresponding value for the Hubble radius (14) and the value we obtained in Sec. III are of the same order of magnitude. This is because the same mass scale (15) is assumed.

Additionally, Milgrom pointed out that $a_{0}$ is also the gravitational acceleration produced by a particle of mass $\sim 100 \mathrm{MeV} / \mathrm{c}^{2}$ at a distance equal to its Compton wavelength and Funkhouser [2] has studied the LNC for the fundamental quantities and the cosmological coincidence (12). He found that there exists a critical acceleration coming from these coincidences and has solved them proposing the following scaling law for the cosmological constant:

$$
\Lambda \approx\left(\frac{8 \pi G}{3 c^{2}}\right) \frac{G m_{n}^{2}}{\lambda_{n}^{4}},
$$

where $m_{n}$ is the nucleon mass and $\lambda_{n}$ is its Compton wavelength. This equation may be interpreted as the energy density associated with the cosmological constant, scaled to the gravitational energy density of the nucleon mass confined to a sphere with its Compton wavelength as the radius.

Evaluating the r.h.s. of the last scaling relation, a discrepancy of $\sim 4$ orders of magnitude is found with respect to the value of the cosmological constant, $\Lambda \approx$ $3.9 \times 10^{-36} \mathrm{~s}^{-2}$. This discrepancy is approximately solved by replacing $\lambda_{n}$ by $b \lambda_{n}$, where $b$ is a constant of order 10 [2].

Assuming the scaling relation (17), and using the Eddington - Weinberg relation, the MOND's acceleration scale is found to be the characteristic gravitational acceleration of the nucleon mass at its Compton wavelength, scaled by the same factor $b=10$, that is

$$
a_{0} \approx \frac{G m_{n}}{\left(b \lambda_{n}\right)^{2}} .
$$

This relation is equivalent to the Milgrom result for a particle of mass $\sim 100 \mathrm{MeV}$, except for a factor $2 \pi$, recovered assuming Eq. (7).

Finally, it is possible to show how to obtain the coincidence relation (12) from the quantization relation (11). Rewriting such a quantum relation in terms of the angular momentum $J$ of the mass $M$, we have

$$
G M^{2}=n \hbar c=J c .
$$

Now, the intrinsic angular momentum for a mass $M$ and a radius $R$ rotating at the angular velocity $\omega$, is given by

$$
J \sim \omega M R^{2} .
$$

Writing the last two relations for the mass and radius of the observed universe, by using Eqs. (20) in (19), and after rearranging terms, we have

$$
\frac{G M_{\mathrm{u}}^{2}}{R_{\mathrm{u}}^{2}} \sim \omega_{0} c \sim \frac{c}{T_{0}},
$$

where $\omega_{0}$ represents the 'intrinsic angular velocity' of the universe and $T_{0}$ its 'rotation period', that can be approximated by the age of the universe. Then, from the Hubble constant, $H_{0} \sim 1 / T_{0}$, and from the scaling relation (8), where $a_{*}=a_{0}$, we recover the coincidence given by Eq. (12).

An intrinsic angular momentum for the entire universe from which this coincidence can be derived, may have some implication for the so-called "axis of evil" [21].

\section{DISCUSSION AND CONCLUSIONS}

In this paper, we have discussed some quantum-cosmic coincidence relations involving the phenomenological parameter $a_{0}$ which sets the so-called MOND scale.

In this perspective, such an acceleration seems more than only a phenomenological parameter, but a fundamental quantity related to some universal constants (Eq. (10) ) and coming from a quantization condition (10) for quantum-gravitational systems.

From the identification $a_{*}=a_{0}$, the scaling relations (6) and (5) hold for any self-gravitating astrophysical structure where gravity is the overall interaction that bounds the system. Such relations connect the microscopic constituents (protons) with the macroscopic features of the astrophysical systems (radius and mass); the acceleration $a_{0}$ gives the natural cut-off where dynamics changes regime without invoking any dark matter. Being connected also with the Hubble parameter $H_{0}$, Eq.(12), and the cosmological constant $\Lambda$, Eq.(17), $a_{0}$ could give rise also to a natural explanation for dark energy phenomena. As a final comment, we have to say that the relations presented here should be seriously considered in view to explain the universe content just with observable quantities. However, stating that $a_{0}$ is a fundamental parameter, we do not pretend to be conclusive since we need a self-consistent relativistic quantum field theory where MOND is fully recovered in the weak field limit.

\section{ACKNOWLEDGEMENTS}

TB acknowledges economic support from CONACyT 207529. 
[1] A.S. Eddington, Proc. of the Cambridge Philosophical Society, 27, 15 (1931).

[2] S. Funkhouser, Proc. R. Soc. A, 462, 3657 (2006).

[3] F. Zwicky, Ap. J., 86, 217 (1937).

[4] J. Binney and S. Tremaine, Galactic Dynamics, Second Edition. Princeton University Press, Princeton (2008).

[5] A. Riess et al., Astr. J., 116, 1009 (1998).

[6] S. Perlmutter et al., Ap. J., 517, 565 (1999).

[7] S. Capozziello and V. Faraoni, Beyond Einstein Gravity, Fundamental Theories of Physics Vol. 170, Springer Ed., Dordrecht (2011).

[8] S. Mendoza, X. Hernandez, J. C. Hidalgo, and T. Bernal, Mon. Not. R.Astr. 411, 226 (2011).

[9] M. Milgrom, Ap. J., 270, 365 (1983).

[10] M. Milgrom, Talk presented at the XIX Rencontres de Blois Matter and energy in the Universe: from nucleosynthesis to cosmology, arXiv:0801.3133 [astro-ph].

[11] M.Milgrom, Phys. Rev., D 80:123536, 0912.0790 (2009).

[12] S. Capozziello, G. Cristofano, M. De Laurentis, Eur.
Phys. J. C 69, 293 (2010).

[13] S. Capozziello, G. Cristofano, M. De Laurentis, to appear in Mod. Phys. Lett. A (2011) arXiv:1110.1175 [gr-qc] .

[14] S. Weinberg, Gravitation and Cosmology, Wiley, New York (1972).

[15] S. Capozziello and S. Funkhouser, Mod. Phys. Lett. A, 24, 1121 (2009).

[16] S. Capozziello and S. Funkhouser, Mod. Phys. Lett. A, 24, 1743 (2009).

[17] P.A.M. Dirac, Proc. Roy. Soc. A 133, 60 (1931).

[18] S. Capozziello, S. De Martino, S. De Siena, F. Illuminati, Mod. Phys. Lett. A 15, 1063 (2000).

[19] A.K. Raychaudhuri, Theoretical Cosmology, Clarendon, Oxford (1979).

[20] K.G. Begeman, A.H. Broeils, R.H. Sanders, Mon. Not. R. Astr. Soc., 249, 523B (1991).

[21] K. Land and J. Magueijo, Mon. Not. R. Astr. Soc., 378, 153 (2007). 